\title{
OCORRÊNCIA DE SALMONELLA SPP. E STAPHYLOCOCCUS COAGULASE POSITIVA EM PESCADO NO NORDESTE, BRASIL
}

\section{D.A.M. Duarte, A.R. Ribeiro, A.M.M. Vasconcelos, J.V.D. Silva, P.L.A. de Andrade, A.A.P. Santana}

Ministério da Agricultura, Pecuária e Abastecimento, Laboratório Nacional Agropecuário, Rua Dom Manoel de Medeiro, s/nº, CEP 52171-030, Recife, PE, Brasil. E-mail: mic-lanagro-pe@agricultura.gov.br

\author{
RESUMO
}

\begin{abstract}
O presente estudo foi conduzido com o objetivo de verificar a ocorrência de Salmonella spp. e Staphylococcus coagulase positiva em pescados e crustáceos provenientes da região nordeste no período de fevereiro de 2004 a dezembro de 2005. Das 143 amostras de peixes e crustáceos (camarão congelado e cauda de lagosta), 5 (3,5\%) apresentaram-se positivas para Salmonella spp. Enquanto nas amostras de peixe e cauda de lagosta a contagem de Staphylococcus coagulase positiva foi $<1,0$ $\times 10^{2} \mathrm{UFC} / \mathrm{g}$ estimado, nas amostras de camarão congelado, em duas a contagem foi $1,0 \times 10^{1} \mathrm{UFC} / \mathrm{g}$ estimado e em uma 2,6 $\times 10^{1} \mathrm{UFC} / \mathrm{g}$ estimado, as demais $<1,0 \times 10^{1} \mathrm{UFC} / \mathrm{g}$ estimado. Com base nos resultados obtidos conclui-se que as amostras analisadas apresentaram baixos níveis de ocorrência de Salmonella spp. e Staphylococcus coagulase positiva e que, mesmo com estes baixos índices, constantemente deve-se buscar uma melhora na qualidade dos produtos da pesca, melhora esta que passa pelo treinamento constante em boas práticas de fabricação, procedimentos padrões de higienização e análise de perigos e pontos críticos de controle do pessoal que trabalha na pesca ou em fazendas de criação de camarão, bem como dos funcionários das indústrias.
\end{abstract}

PALAVRAS-CHAVE: Salmonella spp., Staphylococcus coagulase positiva, peixe, crustáceo.

\section{ABSTRACT}

OCCURRENCE OF SALMONELLA SPP. AND COAGULSE POSITIVE STAPHYLOCOCCUS IN FISH AND CRUSTACEANS IN THE NORTHEAST OF BRAZIL. The present study was carried out to evaluate the occurrence of Salmonella spp. and coagulase-positive Staphylococcus in fish and crustaceans from the Brazilian Northeast. Five out of the 143 samples (3.5\%) were positive for Salmonella spp. While none of the fish and lobster tail samples were positive for coagulase positive Staphylococcus, two shrimp samples presented an estimated count of $1.0 \times 101$ colony forming units per gram $(\mathrm{cfu} / \mathrm{g})$, and one presented $2.6 \times 101 \mathrm{cfu} / \mathrm{g}$. The results showed a low level of Salmonella and coagulase positive Staphylococcus occurrence. However, even with these low levels of bacterial numbers in fish and crustaceans we should search look for an improvement in their quality. This improvement should be associated to constant training for good manufacturing practice, a standard procedure of hygiene and analysis of hazards and critical control points related to employees that work as fishers or on shrimp farms, as well as those working in the industry as a whole.

KEY WORDS: Salmonella, coagulase positive Staphylococcus, fish, crustaceans.

Desde os tempos mais remotos os pescados fazem parte da dieta alimentar do homem (FOOD AND AGRICULTURE ORGANIZATION, 1997) e, atualmente, cada vez mais pessoas preferem consumi-los comoalternativa mais saudável, em relação à carne bovina, suína e de frango, uma vez que são de fácil digestibilidade, possuem elevados níveis de vitaminas A, E e D, possuem grande quantidade de fósforo eiodo (peixes do mar) e são produtos que podem ser indicados para pessoas de qualquer idade (LEDERLE, 1991).

Apesar da extensa costa marítima e da abundância de bacias hidrográficas que recortam o território nacional, no Brasil o consumo de pescado é baixo.
Assim como as carnes de bovinos, de suínos e de frango, os produtos da pesca (peixes e crustáceos) também podem ser veiculadores de uma grande quantidade de micro-organismos patogênicos para o homem, muitas vezes fruto de contaminação ambiental, devido a despejos de esgoto nas águas dos rios, lagos e mar, comprometendo assim a sua qualidade (CONSTANTINIDO, 1994).

Além da contaminação ambiental, as condições higiênico-sanitárias durante a captura e manuseio (ZICAN, 1994), a qualidade do gelo utilizado na conservação e os recipientes onde são transportados ou acondicionados (GERMANO et al., 1998) são fontes 
de contaminação de extrema importância para os produtos da pesca.

Dentre os micro-organismos que podem ser veiculados pelos pescados e crustáceos, destacamse Vibrio spp., Salmonella spp. e Listeria spp. Além dessa microbiota, ocorrem como consequência da manipulação inadequada o Streptococcus spp. e o Staphylococcus aureus, ambos de origem humana, encontrados na pele e mucosa (FORSYTHE, 2002).

O gênero Salmonella, constituído por 2.532 sorovares (Popoff et al., 2003), tem como seu habitat natural o trato intestinal de humanos e animais (DOYLE; Cliver, 1990).

Os alimentos de origem animal, a água, bem como alimentos de origem vegetal têm sido identificados como veículos de transmissão deste micro-organismo (DOYLE; Cliver, 1990).

O S. aureus é uma bactéria capaz de produzir enterotoxinas, as quais são responsáveis pelas intoxicações alimentares (FORSYTHE, 2002).

Toxinfecções alimentares envolvendo $S$. aureus e pescados têm sido relatadas. No Reino Unido, 7\% dos casos de toxinfecções alimentares causadas por S. aures, relatadas no período entre 1969 e 1990, tiveram como veiculador do agente, peixes e mariscos (WiENEKE et al., 1993). Na França, nos anos de 1999 e $2000,11 \%$ dos casos tiveram peixes e frutos do mar como alimento envolvido (HAEGHEBAERT et al., 2002, apud LE LoIR et al., 2003).

O presente estudo foi conduzido com o objetivo de verificar a ocorrência de Salmonella spp. e Staphylococcus coagulase positiva em produtos de pesca provenientes da região Nordeste.

No período de fevereiro de 2004 a dezembro de 2005 , foram analisadas 143 amostras de pescado: peixe (n:50), crustáceos e camarão congelado (n:71) e cauda delagosta (n:22), provenientes dos Estados da Região Nordeste do Brasil, utilizando-se as técnicas de pesquisa de Salmonella spp. e contagem de Staphylococcus coagulase positiva, recomendadas pelo Ministério da Agricultura, Pecuária e Abastecimento (BRASIL, 2003).

Das 143 amostras analisadas, 5 (3,5\%) apresentaram-se positivas para Salmonella spp. Este resultado foi menor que os $6,9 \%$ obtidos por Heinitzet al.(2000), em amostras de pescado, crustáceos e outras criaturas aquáticas que foram importados ou produzidas no Estados Unidos e que os $13,6 \%$ apresentados por KUMAR et al. (2003), em amostras de peixe, camarão e mariscos coletados em Mangalore, Índia.

Na Tabela 1 consta a ocorrência de Salmonella entre os produtos analisados. As amostras de cauda de lagosta tiveram a maior ocorrência $(4,5 \%)$. As amostras de peixe apresentaram Salmonella em $4,0 \%$, fato este que contrasta com os $11,8 \%$ obtidos por HeInitz et al. (2000), em amostras de peixe produzidos e importados pelos Estados Unidos, contudo nas de camarão congelado a ocorrência foi de 2,8\%.
Os dados obtidos para camarão congelado, foram maiores que os 1,6\% encontrados por KoONSE et al. (2005), em camarões provenientes de carcinicultura.

Tabela 1 - Ocorrência de Salmonella spp. em peixes e crustáceos produzidos na região Nordeste.

\begin{tabular}{lc}
\hline Amostra & $\begin{array}{c}\text { Presença/No de } \\
\text { amostras }(\%)\end{array}$ \\
\hline Peixe & $2 / 50(4,0)$ \\
Camarão congelado & $2 / 71(2,8)$ \\
Cauda de lagosta & $1 / 22(4,5)$ \\
\hline Total & $5 / 143(3,5)$ \\
\hline
\end{tabular}

A contaminação por Salmonella spp., quando presente, em produtos da pesca e crustáceos pode ser proveniente da contaminação do ambiente de onde os mesmos foram retirados (MoHAMEd HatHa et al., 2003), ou provenientes de manipulação na despesca e no processamento (Kumar et al., 2003).

Quanto ao Staphylococcus coagulase positiva, este agente etiológico, esteve presenteem 3(2,1\%) das 143 amostras analisadas. A ocorrência de Staphylococcus coagulase positiva se deu nas amostras de camarão congelado, sendo que duas apresentaram como resultado $1,0 \times 10^{1} \mathrm{UFC} / \mathrm{g}$ e uma $2,6 \times 10^{1} \mathrm{UFC} / \mathrm{g}$. As demais amostras de camarão congelado apresentaram como resultado valor $<1,0 \times 10^{1} \mathrm{UFC} / \mathrm{g}$, entretanto as amostras de peixe e cauda de lagosta, os resultados encontrados foram $<1,0 \times 10^{2} \mathrm{UFC} / \mathrm{g}$.

Por meio dos dados obtidos para Staphylococcus coagulase positiva conclui-se que os mesmos encontravam-se de acordo com os padrões estabelecidos para amostras indicativas da Resolução da Diretoria Colegiada (RDC) n 12 , regulamento técnico sobre padrões microbiológicos para alimentos (BRASIL, 2001), porém como o Staphylococcus aureus é um indicador das condições de higiene e sanitização, quando presente em alimento pode indicar que durante o processamento e estocagem tenha ocorrido algum tipo de falha de manipulaçãoe/ou estocagem inadequada e/ou contaminação cruzada (SIMON; SANJEEV, 2007).

Com base nos resultados obtidos conclui-se que as amostras analisadas apresentaram baixos níveis de ocorrência de Salmonella spp. e Staphylococcus coagulase positiva, e que mesmo com estes baixos índices constantemente deve-se buscar uma melhora na qualidade dos produtos da pesca, melhora esta que passa pelo treinamento constante em boas práticas de fabricação, procedimentos padrões de higienização e análise de perigos e pontos críticos de controle do pessoal que trabalha na pesca ou em fazendas de criação de camarão, bem como dos funcionários das indústrias. 


\section{REFERÊNCIAS}

BRASIL. Ministério da Saúde. Resolução - RDC n 12, de 2 de janeiro de 2001. Regulamento técnico sobre padrões microbiológicos para alimentos. Diário Oficial [da] República Federativa do Brasil, Poder Executivo, Brasília, DF, 2 de janeiro de 2001. Seção I, p. 45-53.

BRASIL. Ministério da Agricultura, Pecuária e Abastecimento. Instrução Normativa 62, de 26 de agosto de 2003. Métodos analíticos oficiais para análises microbiológicas para o controle de produtos de origem animal e água. Diário Oficial [da] República Federativa do Brasil, Poder Excutivo, Brasília, DF, 18 de setembro de 2003. Seção I, p.4-50.

CONSTANTINIDO, G. A saúde do pescado depende diretamente da saúde do ambiente. Revista Higiene Alimentar, v.8, n.32, p.5-6, 1994.

DOYLE, M.P.; CLIVER, D.O. Salmonella. In: CLIVER, D.O. (Ed.). Foodborne Diseases. London: Academic Press, 1990. p.185-201.

FOOD AND AGRICULTURE ORGANIZATION. Documento técnico sobre pesca. Garantia da qualidade dos produtos da pesca. 1997. 176p.

FORSYTHE, S.J. Microrganismos causadores de doenças de origem alimentar. In: (Ed.). Microbiologia da segurança alimentar. Porto Alegre: Artmed, 2002. 424p. Cap.5.

GERMANO, P.M.L.; GERMANO, M.I.S.; OLIVEIRA, C.A.F. Aspectos da qualidade do pescado de relevância em saúde pública. Higiene Alimentar, v.12, p.30-37, 1998.

HEINITZ, M.; RUBLE, R.D.; WAGNER, D.E.; TATINI, S.R. Incidence of Salmonella in fish and seafood. Journal of Food Protection, v.63, n.5, p.579-592, 2000.

KOONSE, B.; BURKHARDT III, W.; CHIRTL, S.; HOSKIN, G. Salmonella and sanity quality of aquacultured shrimp. Journal of Food Protection, v.68, p.2527-2532, 2005.
KUMAR H.S.; SUNIL, R.; VENUGOPAL, M.N.; KARUNASAGAR, I.; KARUNASAGAR, I. Detection of Salmonella spp. in tropical seafood by polymerase chain reaction. International Journal Food Microbiology, v. 88, p.91-95, 2003.

LEDERLE, J. Enciclopédia moderna de higiene alimentar. São Paulo: Manole Dois, 1991. 224p.

LE LOIR, Y.; BARON, F.; GAUTIER, M. Sathapylococcus aureus and food Poisoning. Genetics and Molecular Research, v.2, p.63-76, 2003.

MOHAMED HATHA, A.A.; MAQBOOL, T.K.; KUMAR, S.S. Microbial quality of shrimp products of export trade produced from aquacultures shrimp. International Journal Food Microbiology, v.82, p.213-221, 2003.

POPOFF, M.Y.; BOCKEMÜHL, J.; GHEESLING, L.L. Supplement 2001(no. 45) to the Kauffamann-White scheme. Research in Microbiology, v.154, p.173-174, 2003.

SIMON, S.S.; SANJEEV, S. Prevalence of enterotoxigenic Staphylococcus aureus in fishery products and fish processing factory workers. Food Control, v.18, p.15651568. 2007.

WIENEKE, A.A.; ROBERTS, D.; GILBERT, R.J. Stapylococcal food poisoning in the United Kindom, 1969 - 1990. Epidemiology and Infection, v.110, p.519-531, 1993.

ZICAN, C.A. O Ministério da Agricultura iniciou o controle sanitário através do sistema de pontos críticos. $\mathrm{O}$ pescado é o carro chefe desse sistema. Higiene Alimentar, v.8, n.31, p.9-10, 1994. Trabalho apresentado no SEMINÁRIO DE VIGILÂNCIA SANITÁRIA PESQUEIRA: QUALIDADE DOS PESCADOS, 1., 1994, São Paulo. Resumos.

Recebido em 17/2/09

Aceito em 28/10/10 